\title{
Prevalence of biofilm and beta-lactamase producing Staphylococcus in nasal and throat isolates from healthy volunteers: A medical alert
}

\author{
Devapriya $F^{1 *}$ SajithP, ${ }^{1}$ Ranganathan $R,{ }^{1}$ Shanmugam $J^{2}$
}

${ }^{1}$ Department of Microbiology, ${ }^{2}$ Former Professor, Department of Microbiology, Gulf Medical University, Ajman, UAE

*Corresponding Author:

F. Devapriya

Lecturer in Microbiology

Gulf Medical University Ajman, UAE

Email id: devapriya31@yahoo.co.in

\begin{abstract}
Background: The Staphylococci species are the predominant inhabitants of the nose and throat as normal flora, they are now becoming a threat due to their capacity to produce biofilm. Biofilm related infections are difficult to be treated unless it is diagnosed early. The aim was to study the prevalence of biofilm and beta-lactamase production in Staphylococcus isolates from nasal and throat mucosa in healthy volunteers.
\end{abstract}

Methods: Staphlococcal isolates were collected from nasal and throat swabs of 100 healthy volunteers at Gulf Medical University and Gulf Medical College Hospital, Ajman, UAE. Staphylococcus aureus or Coagulase Negative Staphylococcus (CoNS) was classified from growth on Mannitol Salt Agar and tube coagulase test. Biofilm production detected by Christensen's tissue culture plate and Congo red agar methods. Betalactamase production among biofilm producers identified by iodometric tube method.

Results: Among 100 Staphylococcus isolates, 41 were Staphylococcus aureus of which $61 \%$ were biofilm producers of which $46.3 \%$ were betalactamase positive. Among the 59 CoNS isolates, $64.4 \%$ were biofilm producers of which $30.5 \%$ were beta-lactamase positive. Predominance of nasal Staphylococcal isolation observed among males at the age group of $<20$ years.

Conclusion: Biofilm-producing Staphylococcus inhabits the nasal and throat mucosa of healthy individuals. Beta-lactamase production was higher in Staphylococcus aureus positive for biofilm producers as compared to CoNS. Transmission of these biofilm producers with drug resistance factors from the healthy individuals to high risk patients with indwelling devices need to be considered.

Keywords: Beta-lactamase; biofilm; coagulase negative staphylococcus; staphylococcus aureus.

\section{Introduction}

Staphylococcus epidermidis and Staphylococcus aureus are the predominant and persistent inhabitants in the anterior nares and the throat as part of the normal flora. ${ }^{1-3}$ The nasal site serves as a breeding ground for the Staphylococcus species to grow and multiply remaining as non-pathogens, until they disseminate through the blood stream or breached epithelial surface to other sites. ${ }^{4}$ There the growth and up regulation of adherence factors occur. ${ }^{5,6}$

The virulence of the coagulase negative Staphylococcus 
species (CoNS) is related to its capacity to produce biofilms. Such biofilm-related infections are extremely difficult to treat and have to be diagnosed early. The major components of the extracellular polymeric substance (EPS) of the Staphylococcal biofilm consist of poly-N-acetyl glucosamine (PNAG). Certain strains lack PNAG and among these strains the extracellular teichoic acid was noticed as a new component of Staphylococcal biofilm. ${ }^{7}$ Formation of biofilm in Staphylococcus is considered a four step process, consisting of adherence, accumulation, maturation and later dispersal. $^{7}$

Biofilm is an important colonization factor as well as a virulence factor in bacterial adherence., ${ }^{4-812}$ Colonization occurs in the principal implants like central venous catheters, heart valves, ventricular assist devices, coronary stents, neurosurgical ventricular shunts, implantable neurological stimulators, fracture-fixation devices, arthro-prostheses, inflatable penile implants, breast implants, cochlear implants, intra ocular lenses and dental implants. ${ }^{13-16}$ The objective of this study was to determine the presence of biofilm formation and beta-lactamase production in Staphylococcus aureus and CoNS inhabiting as the normal flora in the anterior nares and throat of normal healthy individuals belonging to a Medical University.

\section{Methods}

The study was conducted among 100 healthy volunteers in Gulf Medical University (GMU), Ajman, UAE after getting the approval from the institutional Ethics Committee. Written consent was taken from all the volunteers after explaining the objective of the study and the procedure of taking samples. The nasal and throat swabs were aseptically collected and processed for Gram staining and isolation on Blood agar, Mannitol salt agar and Mac Conkey agar. The Staphylococcus isolates were categorized as Staphylococcus aureus and CoNS based on their cultural characteristics and standard tube coagulase test. ${ }^{17}$ The biofilm qualitative detection was done by two methods: Christensen's method and Congo Red Agar (CRA) method.

In Christensen's method, a few colonies of the test organism were inoculated in $200 \mu$ trypticase soy broth with $1 \%$ glucose in triplicate into flat bottom polystyrene tissue culture plate wells and incubated for 24 and 48 hours at $37^{\circ} \mathrm{C}$ aerobically. The contents were gently aspirated and the wells were washed several times with phosphate buffer saline with a $\mathrm{pH}$ of 7.2 to remove the free floating bacteria. The biofilm formed in the microwells were stained with $1 \%$ neutral red. The biofilm positive bacteria stained pink at the bottom and on the walls of the tissue culture plate wells [18]. ${ }^{18}$

In CRA method, as described by Freeman et al. the CRA was prepared with brain heart infusion broth $37 \mathrm{~g} / \mathrm{L}$, sucrose $50 \mathrm{~g} / \mathrm{L}$, agar $10 \mathrm{~g} / \mathrm{L}$, and Congo red indicator $8 \mathrm{~g} / \mathrm{L}$. Sterile Congo red stain was prepared as a concentrated aqueous solution. It was added to the sterilized brain heart infusion agar with sucrose at $55^{\circ} \mathrm{C}$. The CRA plates were inoculated by the streak method with the test organisms and incubated at $37^{\circ} \mathrm{C}$ for 24 hours and after checking incubation was continued for another 24 hours aerobically. Isolates positive for biofilm production formed black colored dry colonies of a crystalline consistency and biofilm negatives produced pink colored smooth colonies. ${ }^{19-21}$

Further, these isolates were screened for beta-lactamase by the Iodometric tube method (ITM).The beta-lactamase positive isolates were screened for antibiotic susceptibility testing by the standard Kirby Bauer disc diffusion method..$^{22}$

\section{Results}

The male to female ratio among the 100 healthy volunteers was 54:46. The gender participation in various age groups is shown in the Figure1. The age distribution was between 18 and 60 years. The majority of the participants $(85 \%)$ were below 30 years of age.

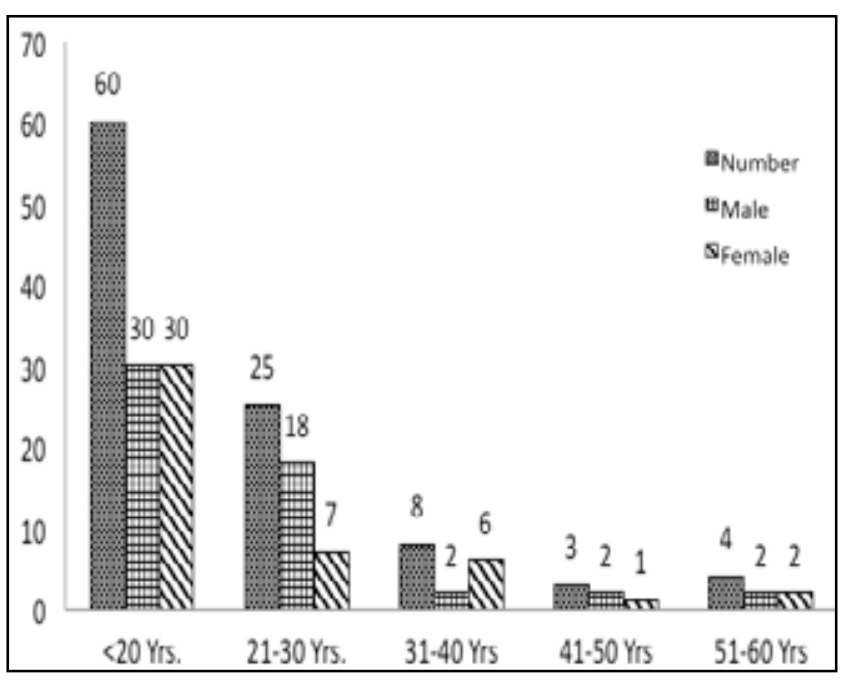

Figure 1. Gender participation ratios with age

Of the 100 nasal swabs collected 63 isolated Staphylococcus species, 24 were Gram positive bacilli (Diphtheroids) and 13 swabs had no growth. Out of 100 throat swabs 37 isolated Staphylococcus species, 2 Gram negative bacilli (Klebsiella pneumonia and Enterobacter sp.), 41 Gram negative cocci in groups (non- pathogenic Neisseria) and 20 swabs showed no growth.

Staphylococcal isolation was 37\% from the throat and higher in the nasal site $(63 \%)$ of which $21 \%$ were Staphylococcus aureus. Isolation rate was higher in the age group below 20 years of age (58\%), followed by the $21-30$ years $(26 \%)$. In the remaining age groups isolation was minimal.

As shown in Table 1, Irrespective of age and gender, the CoNS isolates were significantly higher than Staphylococcus aureus in nasal and throat specimen (3:2). The number of Staphylococcus isolates was common among the younger age group ( $<20$ years). 
Table 1.Distribution of Staphylococcus isolates based on age and gender

\begin{tabular}{lccccc}
\hline \multirow{2}{*}{$\begin{array}{c}\text { Age in } \\
\text { Years }\end{array}$} & \multicolumn{2}{c}{ Male $\mathbf{n}=\mathbf{5 4}$} & \multicolumn{3}{c}{ Female $\mathbf{n}=\mathbf{4 6}$} \\
\cline { 2 - 6 } & S.aureus & CoNS & S.aureus & CoNS & Total \\
\cline { 2 - 6 } & No. & No. & No. & No. & No. \\
\hline$<20$ & 13 & 20 & 10 & 15 & 58 \\
$21-30$ & 08 & 06 & 02 & 10 & 26 \\
$31-40$ & 02 & 01 & 02 & 04 & 08 \\
$41-50$ & 01 & 01 & 01 & 0 & 04 \\
$51-60$ & 0 & 01 & 01 & 01 & 03 \\
$>60$ & 01 & 0 & 0 & 0 & 01 \\
\hline Total & 25 & 29 & 16 & 30 & 100 \\
$\%$ & 46.2 & 53.7 & 34.7 & 65.2 & \\
\hline
\end{tabular}

Table 2.Biofilm and Beta-lactamase production in Staphylococcus isolates

\begin{tabular}{|c|c|c|c|c|c|c|}
\hline \multirow[t]{2}{*}{ Methods } & \multicolumn{2}{|c|}{$\begin{array}{c}\text { Congo } \\
\text { Red Agar } \\
\text { Method } \\
\text { (CRA) } \\
\text { Biofilm (+) }\end{array}$} & \multicolumn{2}{|c|}{$\begin{array}{c}\text { Tissue } \\
\text { culture Plate } \\
\text { Method } \\
\text { (TCP) } \\
\text { Biofilm }(+)\end{array}$} & \multicolumn{2}{|c|}{$\begin{array}{c}\text { Tube } \\
\text { Iodometric } \\
\text { Method (ITM) } \\
\text { Beta- } \\
\text { lactamase }(+)\end{array}$} \\
\hline & No. & $\%$ & No. & $\%$ & No. & $\%$ \\
\hline $\begin{array}{l}\text { Staphylococcus } \\
\text { aureus }\end{array}$ & 25 & 61 & 25 & 61 & 32 & 78 \\
\hline CoNS & 34 & 57.6 & 38 & 64.4 & 22 & 37.2 \\
\hline
\end{tabular}

The presence of the biofilm formation was tested by two methods CRA and TCP methods and Beta-lactamase by ITM. Biofilm detection by both the methods were alike in Staphylococcus aureus (60.9\%),whereas more positivity (64.4\%) was obtained by the TCP method compared to the CRA method (57.6\%) in the CoNS. Betalactamase was positive for Staphylococcus aureus (78\%) and positive for CoNS (37.2\%)as shown in the Table 2.

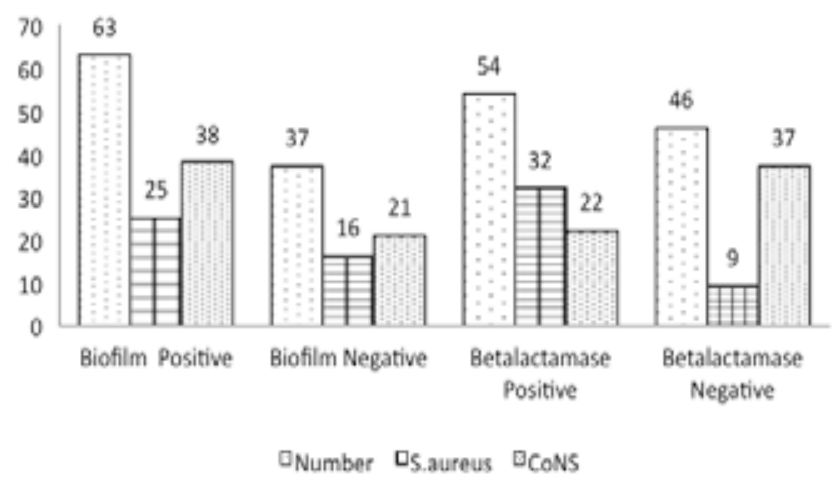

Figure 2. Biofilm production by Tissue Culture Plate method and Beta-lactamase production by Tube Iodometric Method in Staphylococcus isolates

Figure 2 shows the number of positive and the negative results for biofilm production by both the tissue culture plate method and beta-lactamase production by Tube iodometric method among the Staphylococcus aureus and CoNS. Of the 100 Staphylococcus isolates 37 were negative for biofilm production and 46 were negative for betalactamase production. The beta-lactamase positive isolates were resistant to Penicillin and Ampicillin and susceptible to Vancomycin, Oxacillin and Rifampicin.

\section{Discussion}

Biofilm formation in bacteria is now a major problem in the medical field since it is responsible for many recalcitrant infections and also difficult to be eradicated. It contributes to virulence factors like the ability to avoid host immune response, restricted penetration of antimicrobial agents into the biofilm and exhibition of resistance to antibiotics due to various mechanisms including beta-lactamase production.

Staphylococcal isolation was high in the nasal site among the Japanese population, as reported by Tadayukiin. ${ }^{23}$ Our results were comparable, with increased Staphylococcus isolation $(62 \%)$ from the nasal site. Karina et al. conducted a similar study among the medical students in Brazil and observed a percentage of nasal Staphylococcus aureus isolation of $40.8 \%{ }^{24}$ In our study the nasal Staphylococcus aureus isolation was $21 \%$. Samie et al. conducted a similar study on biofilm and beta-lactamase detection using similar methods and detected $42 \%$ were biofilm producers among which $16 \%$ were beta- lactamase positive..$^{25}$ In our study, $63 \%$ were biofilm producers among which $37 \%$ were betalactamase positive. Our study detected $63 \%$ of biofilm producing Staphylococcus isolates, similar to a study done in Pakistan (54.8\%) by Joanna et al. ${ }^{26}$ The resemblance in the results may be due to similar culture, living conditions and geographical location. Both the conventional methods for biofilm detection (CRA and TCP) gave similar results in Staphylococcus aureus isolates, whereas the positivity in CoNS was slightly higher by the TCP method, agreeing with the earlier studies. ${ }^{27-28}$ However, Ruzicka et al. had done genetic studies detecting ica operon responsible for the biofilm production and compared with the similar conventional methods adopted in this study. ${ }^{28}$ The results had higher correlation with Christensen's method than with the CRA method. ${ }^{27}$ Our findings differ from the earlier reports where the authors had suggested the CRA method to be superior to TCP. ${ }^{22,30,31}$

Colonization of biofilm forming CoNS is the current threat to effective antibiotic therapy because of the increasing difficulty in detection and management of infections, leading to fatal outcomes. All the beta-lactamase producers were resistant for Penicillin and Ampicillin but showed $100 \%$ sensitivity to Vancomycin corresponding with the earlier reports. ${ }^{24}$

There is a possibility of transmission of these virulence factors from the healthy individuals to those at high risk such as patients on long term catheterization, or having indwelling devices in a medical set up, which may be difficult to treat with the commonly available antimicrobial agents. 


\section{Conclusion}

Biofilm detection is more reliable by the Tissue culture plate method than with the Congo Red Agar method. Also, the virulence factors, biofilm production and beta-lactamase production, seem to be present in the Staphylococcus isolates which normally inhabit the upper respiratory tract.

Since the biofilm and beta-lactamase virulence factors seem to be present in the normal flora of healthy individuals there is a need to screen for them among the healthy individuals who are posted at high risk units where medical device implantation, catheterization etc. are commonly carried out.

\section{Acknowledgement}

The authors express their gratitude to all the volunteers who gave support towards the study, Prof. Anoop Agarwal for his editorial guidance, the management of Gulf Medical University and the Department of Microbiology for the encouragement and support.

Conflict of interest: The authors declare that they have no conflict of interest.

Funding: None

\section{References:}

1. Wesley EK, Margaret SMW. Distribution and Persistence of Staphylococcus and Micrococcus species and other Aerobic Bacteria on Human Skin, App Microbiol 1975;30:381-95.

2. Klytmans J, Van BA, Verbrugh H. Nasal carriage of Staphylococcus aureus: Epidemiology, underlying mechanisms and associated risks. ClinMicrobiol Rev 1997; 10:505-20.

3. Dall' Antonia $\mathrm{M}$, Coen $\mathrm{PG}$, Wilks $\mathrm{M}$, et al. Competition between methicillin -sensitive and resistant Staphylococcus aureus in the anterior nares. J Hosp Infect 2005;61:62-7.

4. Archer NK, Mark JM, Costerton WJ, et al. Staphylococcus aureus biofilms Properties, regulation and roles in human disease. Virulence 2011;2:445-59.

5. Beenken KE, Dunman PM, Aleese MCR, et al. Global gene expression in Staphylococcus aureus biofilm. J Bacteriol 2004;186:4665-84.

6. Fitzpatrick F, Humphreys H, O'Gara JP. The genetics of Staphylococcal biofilm formation-will a greater understanding of pathogenesis lead to better management of device related infections? ClinMicrobiol Infect 2005; 11:967-73.

7. Jabbouri S, Sadovskaya I. Characteristics of the biofilm matrix and its role as a possible target for the detection and eradication of Staphylococcus epidermidis associated with medical implant infections. FEMS
Immunol Med Microbiol 2010;59:280-91.

8. Hall-Stoodley L, Costerton JW, Stoodley P. Bacterial Biofilms: from the natural environment to infectious disease. Rev Microbiol 2004;2:95-108.

9. Costerton JW, Geesey GG, Cheng GK. How bacteria stick. Sci. Am 1978;238:86-95.

10. Costerton JW, Lewandowski Z, Caldwell DE, et al. Microbial biofilms, Annu Rev Microbiol 1995;49:71145 .

11. Simojoki H, Hyvonen P, Ferres CP, et al. Is the biofilm formation and slime production ability of coagulase negative Staphylococci associated with the persistence and severity of intramammary infection? Vetenery Microbiology. 2012. Available from: URL: ELSEVIER. http://dx.doi.org/10.1016/j. vetmic.2012.02.031.

12. Stephen C, Davis BS, Ricotti C et al. Microscopic and physiological evidence for biofilm- associated wound colonization in vivo. Wound Repair and Regeneration 2008;16:23-9.

13. Kaplan JB, Ragunath C, Velliyagounder K, et al. EnzymaticDetachment of Staphylococcus epidermidis Biofilms.AntimicrobagentsChemother 2004;48:26336.

14. Stewarta PS and Costertona JW. Antibiotic resistance of bacteria in biofilms. The Lancet 2001;358:135-8.

15. Christensen GD, Baldassarri L, Simpson WA. Colonization of Medical devices by coagulase negative Staphylococci. In:BisnoAL, Waldvogel FA, Infections associated with indwelling Medical devices. $2^{\text {nd }} e d$. Washington,DC:American Society for Microbiology 1994;45-78.

16. Gristina AG. Biomaterial-centered infection: Microbial adhesion versus tissue integration. Science 1987;23:1588-95.

17. Baird D. Stapylococcus: Cluster-forming Grampositive cocci. Chapter 11: Mackie \& McCartney Practical Medical Microbiology 14th Edition. Singapore: Longman Singapore Publishers;1996.

18. Christenson GD, Simpson WA, Bisno Al, et al. Adherence of slime-producing strains of Staphylococcus epidermidis to smooth surfaces. Infect.Immun 1982;37:318-26.

19. Freeman DJ, Falkiner FR, Keane CT. New method for detecting slime production by coagulase-negative staphylococci; J ClinPathol 1989;42:872-4.

20. Rathinam K, Shanmugam J, Rout D. Slime production by coagulase-negative Staphylococcal species isolated from hospitalized patients, healthy carriers and 
environment. Ind J Med Microbiol 1993;17:243-6.

21. Rathinam K, Shanmugam J. Detecting slime production by Staphylococcus epidermidis strains; Biomed 1995;15:23-6.

22. Miles RS, Amyes SGB. Laboratory control of antimicrobial therapy In: Mackie \& McCartney Practical Medical Microbiology $14^{\text {th }}$ Edition. Singapore: Longman Singapore Publishers;1996.

23. Tadayuki I, Uehara Y, Shinju H, et al. Staphylococcus epidermidisEsp inhibits Staphylococcus aureus biofilm formation and nasal colonization. Nature 2010;465:346-9.

24. Prates KA, Torres AM, Garcia LB, et al. Nasal carriage of methicillin resistant Staphylococcus aureus in university students. Braz J Infect Dis 2010;14:316-8.

25. Samie A, Kgan NTF. Biofilm production and antibiotic susceptibility profile of Eschereichia coli isolates from HIV and AIDS patients in the Limpopo Province. Afr $\mathrm{J}$ of Bacteriol 2012;11:8560-70.

26. Joanna W, Ciok-Pater E, Sekowska A, et al. Comparison of three methods detection of slime production by Staphylococcus aureus and Staphylococcus epidermidis. Mikrobiol 2010;62:303-8.

27. Hassan A, Usman J, Kaleem F, et al. Evaluation of different detection method of biofilm formation in the clinical isolates. Braz J Infect Dis 2011;15:305-11.

28. Ruzicka F, Hola V, Votara M. Biofilm detection and clinical significance of Staphylococcus epidermidis isolates. Folia Microbiol (Praha) 2004;49:596-600.

29. Baqai R, Aziz, M, Rasool G. Urinary tract infection in diabetic patients and biofilm formation of Uropathogens. Infect Dis J Pakistan 2008;17:7-9.

30. Knobloch JK, Kotte MAH, Rohde H, et al. Evaluation of different detection methods of biofilm formation in Staphylococcus aureus. Med Microbial Immunol 2002;191:101-6.

31. Nayak N, Satpathy G, Vajpayee RB, et al. A simple alternative method for rapid detection of slime produced by Staphylococcus epidermidis isolates in bacterial keratitis. Ind J Med Res 2001;114:169-72. 